\title{
ACHIEVABLE RATES OF MULTI-HOP AND COOPERATIVE MIMO AMPLIFY-AND-FORWARD RELAY SYSTEMS WITH FULL CSI
}

\author{
Nicola Varanese*, Osvaldo Simeone*, Yeheskel Bar-Ness \\ *CWCSPR - NJIT \\ \{nv35, osvaldo.simeone $\} @$ njit.edu \\ barness@yegal.njit.edu
}

Umberto Spagnolini

*DEI

Politecnico di Milano

spagnoli@elet.polimi.it

\begin{abstract}
In this paper ${ }^{1}$, two types of MIMO Amplify-and-Forward relay systems are considered: multi-hop and cooperative. For both cases, the problem of maximizing the achievable rate over the covariance matrices of the symbols transmitted by the source and relay linear processing matrix is formulated under the assumption of full channel state information at each node. A sub-optimal iterative algorithm is proposed and proved by numerical simulations to outperform known schemes.
\end{abstract}

\section{INTRODUCTION}

Cooperation is a new paradigm for reliable and high- throughput multi-user wireless communications. The building block of cooperative systems is the relay channel, introduced in [1], where a max-flow-min-cut upper bound for its capacity was derived. To date, the capacity-achieving coding strategy for this channel is still unknown, and simplified cooperative communication schemes have been recently proposed in [2], namely Amplify-and-Forward (AF) and Decode-andForward (DF). The main difference between these cooperative schemes and conventional multi-hop regenerative (DF) or non-regenerative $(\mathrm{AF})$ relaying schemes is that the destination decodes the source message from the signals received from both the source and the relay node. Modified versions of the original DF and AF collaborative protocols, capable of achieving higher rates, have been introduced in [3]. Both [2] and [3] focus on the case where each node is deployed with a single antenna.

The multi-antenna relay channel has been recently investigated from different perspectives. The authors of [4] extended the information-theoretic results of [1] to a multiantenna scenario and devised an algorithm to compute the input covariance matrices that maximize the max-flow-mincut upper bound. Performance of the AF scheme of [2] in a multi-antenna setting was analyzed in [5]. In particular, [5]

\footnotetext{
${ }^{1}$ This work was partially supported by grant from Samsung Electronics Co., LTD.
}
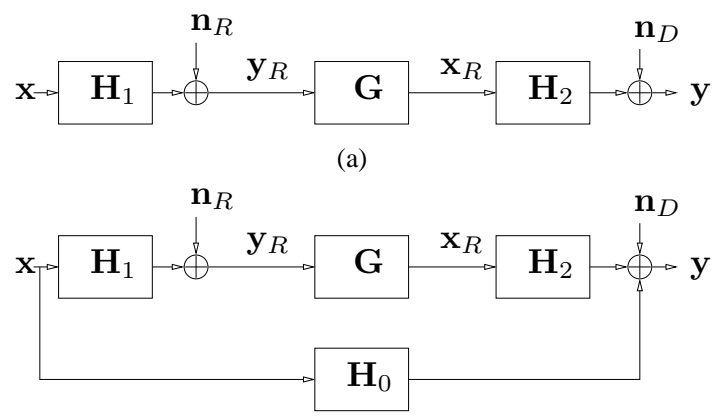

(b)

Fig. 1. Block diagram of a multi-hop (a) and cooperative (b) MIMO Amplify-and-Forward relay system.

derived the optimal linear processing matrix at the relay, for both multi-hop and cooperative MIMO AF relay systems, under the assumption of perfect channel state information (CSI) at each node and isotropic covariance matrix for the symbol transmitted by the source.

In this paper, we consider multi-hop and cooperative MIMO AF relay systems with perfect CSI at each node as in [5]. However, differently from [5]: (i) for both multihop and cooperative systems, the covariance matrix of the symbols transmitted by the source is not constrained to be isotropic; ( $i$ i) for the cooperative scenario, the considered AF protocol is the one presented in [3], whereby the source transmits in both time-slots (not only in the first). The problem of maximizing the achievable rate over the source covariance matrices and linear processing matrix at the relay is formulated. An iterative algorithm, capable of finding a sub-optimal feasible solution, is proposed for both multihop and cooperative cases, and proved by numerical simulations to outperform known schemes under broad channel conditions. 


\section{THE MULTI-HOP MIMO AF RELAY}

\subsection{System model}

The multi-hop MIMO Amplify-and-Forward relay is illustrated in fig.1-(a). In this system three nodes are involved in the communication: a source, a relay and destination, each equipped with $N$ antennas. The operation is divided into two time-slots: in the first time-slot the relay node receives the vector symbol transmitted by the source, while in the second time-slot the relay re-transmits the received vector symbol towards the destination after a linear transformation. The destination decodes based only on the signal received in the second time-slot. All the nodes are assumed to have full CSI. Moreover, the channel matrices are assumed to be independent Rayleigh fading processes, with a coherence time of at least two time-slots and i.i.d. entries.

During the first time slot, the relay receives a signal $\mathbf{y}_{R}=\mathbf{H}_{1} \mathbf{x}+\mathbf{n}_{R}$, where $\mathbf{x}$ is the $N \times 1$ vector transmitted by the source, $\mathbf{H}_{1}$ is the $N \times N$ source-relay channel matrix and $\mathbf{n}_{R}$ is the $N \times 1$ noise vector at the relay node, assumed to have distribution $\mathcal{C N}\left(\mathbf{0}, \sigma^{2} \mathbf{I}\right)$. The relay processes $\mathbf{y}_{R}$ through multiplication by a $N \times N$ matrix $\mathbf{G}$, retransmitting the symbol $\mathbf{x}_{R}=\mathbf{G y}_{R}$ in the second time-slot. Therefore, the input/output relation for the overall system is:

$$
\begin{gathered}
\mathbf{y}=\mathbf{H}_{2} \mathbf{x}_{R}+\mathbf{n}_{D}= \\
=\mathbf{H}_{2} \mathbf{G H}_{1} \mathbf{x}+\mathbf{H}_{2} \mathbf{G n}_{R}+\mathbf{n}_{D},
\end{gathered}
$$

where $\mathbf{y}$ is the received symbol at the destination, $\mathbf{H}_{2}$ is the $N \times N$ relay-destination channel matrix, and $\mathbf{n}_{D}$ is the $N \times 1$ noise vector at the destination, assumed to have distribution $\mathcal{C N}\left(\mathbf{0}, \sigma^{2} \mathbf{I}\right)$.

\subsection{Problem formulation}

In this paper, we are interested in maximizing the mutual information between the source input $\mathrm{x}$ and the destination output $\mathbf{y}, I(\mathbf{x} ; \mathbf{y})$, over the source input covariance matrix $\mathbf{Q}=E\left[\mathbf{x x}^{H}\right]$ and the signal processing at the relay $\mathbf{G}$, under instantaneous power constraints over the source and relay input symbols. Notice that the ergodic achievable rate [6] of the system is $\frac{1}{2} E_{\mathcal{H}}[I(\mathbf{x} ; \mathbf{y})]$, where $E_{\mathcal{H}}[\cdot]$ denotes the average with respect to fading and the factor $1 / 2$ accounts for the two-slots transmission. Thus, we can formulate our optimization problem as

$$
\begin{aligned}
& \max _{\mathbf{Q}, \mathbf{G}} I(\mathbf{x} ; \mathbf{y}) \\
& \text { s.t. }\left\{\begin{array}{l}
\mathbf{Q} \succeq \mathbf{0} \\
\operatorname{tr}(\mathbf{Q})=P \\
\operatorname{tr}(\mathbf{R}(\mathbf{Q}, \mathbf{G}))=P_{R}
\end{array}\right.
\end{aligned}
$$

where

$I(\mathbf{x}, \mathbf{y})=C\left(\mathbf{H}_{2} \mathbf{G H}_{1} \mathbf{Q} \mathbf{H}_{1}^{H} \mathbf{G}^{H} \mathbf{H}_{2}^{H}\left(\sigma^{2} \mathbf{I}+\sigma^{2} \mathbf{H}_{2} \mathbf{G G}^{H} \mathbf{H}_{2}^{H}\right)^{-1}\right)$,

$I(\mathbf{x}, \mathbf{y})=C\left(\mathbf{H}_{2} \mathbf{G} \mathbf{H}_{1} \mathbf{Q} \mathbf{H}_{1} \mathbf{G}^{H} \mathbf{H}^{H}\left(\sigma^{2} \mathbf{I}+\sigma^{2} \mathbf{H}_{2} \mathbf{G G}^{H} \mathbf{H}^{H}\right)\right.$

$$
\mathbf{R}(\mathbf{Q}, \mathbf{G})=E\left[\mathbf{x}_{R} \mathbf{x}_{R}^{H}\right]=\mathbf{G}\left(\mathbf{H}_{1} \mathbf{Q} \mathbf{H}_{1}^{H}+\sigma^{2} \mathbf{I}\right) \mathbf{G}^{H} .
$$

To simplify the notation, we define $C(\mathbf{X}):=\log (\operatorname{det}(\mathbf{I}+$ $\mathbf{X})$ ). According to (2b), the power is fixed to $P$ for the source and to $P_{R}$ for the relay node.

\subsection{An iterative solution}

The optimization problem (2) is not convex. However, if we fix either of the two matrix variables, $\mathbf{Q}$ or $\mathbf{G}$, the resulting problem is convex in the remaining variable. Our solution to the problem (2) is then an iterative procedure that alternates between the optimization over $\mathbf{G}$ fixed $\mathbf{Q}$ and the optimization over $\mathbf{Q}$ fixed $\mathbf{G}$. Absolute convergence to an optimal solution for this algorithm cannot be proved, since the power constraint on the relay input symbol depends upon both $\mathbf{Q}$ and $\mathbf{G}$ [7]. Nevertheless, if the problem is wellconditioned the algorithm has shown in practice rapid convergence (around five iterations) to a unique solution.

Let us start by fixing $\mathbf{G}$. The resulting optimization problem over $\mathbf{Q}$ has a concave objective function and two affine equality constraints. However, as detailed in the following, for the sake of our algorithm, the second constraint can be ignored. It follows that the resulting problem is

$$
\begin{aligned}
& \max _{\mathbf{Q}} C\left(\mathbf{P Q} \mathbf{P}^{H}\right) \\
& \text { s.t. }\left\{\begin{array}{l}
\mathbf{Q} \succeq \mathbf{0} \\
\operatorname{tr}(\mathbf{Q})=P
\end{array},\right.
\end{aligned}
$$

where $\mathbf{P}=\left(\sigma^{2} \mathbf{I}+\sigma^{2} \mathbf{H}_{2} \mathbf{G G}^{H} \mathbf{H}_{2}^{H}\right)^{-\frac{H}{2}} \mathbf{H}_{2} \mathbf{G H}_{1}$ (and the matrix square-root is defined as $\left.\mathbf{F}=\mathbf{F}^{\frac{H}{2}} \mathbf{F}^{\frac{1}{2}}\right)$. Solution of (5) can be found according to [6] by transmitting along the eigenmodes of the equivalent channel $\mathbf{P}$, with power distributed along the sub-channels following the water-filling procedure.

On the other end, if we fix $\mathbf{Q}$, the optimization problem boils down to

$$
\begin{gathered}
\max _{\mathbf{G}} C\left(\mathbf{H}_{2} \mathbf{G A} \mathbf{A}^{H} \mathbf{G}^{H} \mathbf{H}_{2}^{H}\left(\sigma^{2} \mathbf{I}+\sigma^{2} \mathbf{H}_{2} \mathbf{G G}^{H} \mathbf{H}_{2}^{H}\right)^{-1}\right) \\
\text { s.t. } \operatorname{tr}\left(\mathbf{G}\left(\mathbf{A} \mathbf{A}^{H}+\sigma^{2} \mathbf{I}\right) \mathbf{G}^{H}\right)=P_{R},
\end{gathered}
$$

where $\mathbf{A}=\mathbf{H}_{1} \mathbf{Q}^{\frac{H}{2}}$ is the equivalent first-hop channel. This problem has been solved in [5]. Below we briefly summarize the solution by casting it into our notation.

Expanding $\mathbf{H}_{2}$ and $\mathbf{A}$ with the corresponding singular value decomposition $\mathbf{H}_{2}=\mathbf{U}_{2} \boldsymbol{\Lambda}_{2} \mathbf{V}_{2}^{H}$ and $\mathbf{A}=\mathbf{U}_{A} \boldsymbol{\Lambda}_{A} \mathbf{V}_{A}^{H}$, the objective and constraint functions are easily diagonalized by choosing $\mathbf{G}=\mathbf{V}_{2} \mathbf{D}_{g} \mathbf{U}_{A}^{H}$, where $\mathbf{D}_{g}$ is a $N \times N$ diagonal matrix. The solution is similar to a water-pouring over the eigenmodes of the relay-destination channel $\mathbf{H}_{2}$ :

$\left|g_{r}\right|^{2}=\frac{1}{\left(\lambda_{A, r}^{2}+\sigma^{2}\right)}\left[f\left(\mu ; \eta_{r}\right)-\frac{\sigma^{2}}{\lambda_{2, r}^{2}}\right]^{+}$ 


$$
f\left(\mu ; \eta_{r}\right)=\sqrt{\left(\frac{\eta_{r}}{2}\right)^{2}+\eta_{r} \mu^{2}}-\frac{\eta_{r}}{2},
$$

where $[x]^{+}=\max (x, 0) ; \lambda_{A, r}$ and $\lambda_{2, r}$ are the $r$-th singular value of $\mathbf{A}$ and $\mathbf{H}_{2} ; g_{r}$ is the $r$-th diagonal element of the matrix $\mathbf{D}_{g}$ and $\eta_{r}=\frac{\lambda_{A, r}^{2}}{\lambda_{2, r}^{2}}$. The value of $\mu$ is chosen as to satisfy the power constraint on the relay input symbol $\sum_{r=1}^{N}\left(\lambda_{A, r}^{2}+\sigma^{2}\right)\left|g_{r}\right|^{2}=P_{R}$.

Following from this results, we can finally detail our algorithm: (a) solve the optimization problem (5) for $\mathbf{Q}$ keeping $\mathbf{G}$ fixed; (b) solve the optimization problem (6) for $\mathbf{G}$ keeping $\mathbf{Q}$ fixed. Notice that the last iteration of the algorithm has to be (6), since this guarantees the enforcement of all the constraints in the original problem (2).

\section{THE COOPERATIVE MIMO AF RELAY}

\subsection{System model}

The cooperative MIMO Amplify-and-Forward relay is illustrated in fig.1-(b). In the first time-slot the source transmits a first signal $\mathbf{x}_{1}$ to both the relay and the destination; in the second time-slot the relay retransmits $\mathbf{x}_{1}$ after a linear transformation, while the source transmits a second signal $\mathrm{x}_{2}$, independent from $\mathbf{x}_{1}$. At the end of the second time-slot, the destination jointly decodes $\left(\mathbf{x}_{1}, \mathbf{x}_{2}\right)$ from the signals received in the two time-slots.

The signal received by the destination node during the first time-slot is $\mathbf{y}_{1}=\mathbf{H}_{0} \mathbf{x}_{1}+\mathbf{n}_{D, 1}$, where $\mathbf{x}_{1}$ is the $N \times 1$ source input symbol, $\mathbf{H}_{0}$ is the $N \times N$ source-destination channel, and $\mathbf{n}_{D, 1}$ is the $N \times 1$ noise vector at the destination. During the same time-slot, the relay node receives a signal $\mathbf{y}_{R}=\mathbf{H}_{1} \mathbf{x}_{1}+\mathbf{n}_{R, 1}$, where $\mathbf{H}_{1}$ is the $N \times N$ sourcerelay channel and $\mathbf{n}_{R, 1}$ is the $N \times 1$ noise vector at the relay node. The relay processes $\mathbf{y}_{R}$ through multiplication by a $N \times N$ matrix $\mathbf{G}$, retransmitting the symbol $\mathbf{x}_{R}=\mathbf{G y}_{R}$ in the second time-slot. At the same time, the destination transmits a new symbol $\mathbf{x}_{2}$, independent from $\mathbf{x}_{1}$. Therefore, during the second time-slot the destination receives a symbol $\mathbf{y}_{2}=\mathbf{H}_{0} \mathbf{x}_{2}+\mathbf{H}_{2} \mathbf{x}_{R}+\mathbf{n}_{D, 2}$, where $\mathbf{H}_{2}$ is the relaydestination channel and $\mathbf{n}_{D, 2}$ is the $N \times 1$ noise vector at the destination. Finally, the overall input/output relation for this communication scheme is

$$
\left[\begin{array}{l}
\mathbf{y}_{1} \\
\mathbf{y}_{2}
\end{array}\right]=\left[\begin{array}{cc}
\mathbf{H}_{0} & \mathbf{0} \\
\mathbf{H}_{2} \mathbf{G H}_{1} & \mathbf{H}_{0}
\end{array}\right]\left[\begin{array}{c}
\mathbf{x}_{1} \\
\mathbf{x}_{2}
\end{array}\right]+\mathbf{n}_{e q}
$$

where we have defined the equivalent noise vector as

$$
\mathbf{n}_{e q}=\left[\begin{array}{ccc}
\mathbf{I} & \mathbf{0} & \mathbf{0} \\
\mathbf{0} & \mathbf{H}_{2} \mathbf{G} & \mathbf{I}
\end{array}\right]\left[\begin{array}{c}
\mathbf{n}_{D, 1} \\
\mathbf{n}_{R, 1} \\
\mathbf{n}_{D, 2}
\end{array}\right] .
$$

Since each noise vector is assumed to have a distribution $\mathcal{C N}\left(\mathbf{0}, \sigma^{2} \mathbf{I}\right)$, the equivalent noise correlation matrix
$\mathbf{C}=E\left[\mathbf{n}_{e q} \mathbf{n}_{e q}^{H}\right]$ is
$\mathbf{C}=\left[\begin{array}{cc}\sigma^{2} \mathbf{I} & \mathbf{0} \\ \mathbf{0} & \sigma^{2} \mathbf{I}+\sigma^{2} \mathbf{H}_{2} \mathbf{G G}^{H} \mathbf{H}_{2}^{H}\end{array}\right]=\left[\begin{array}{cc}\sigma^{2} \mathbf{I} & \mathbf{0} \\ \mathbf{0} & \mathbf{C}_{2}(\mathbf{G})\end{array}\right]$.

Assuming for the sake of simplicity that $\mathbf{x}_{1}$ and $\mathbf{x}_{2}$ are independent, the input covariance matrix is block diagonal:

$$
E\left[\mathbf{x x}^{H}\right]=\mathbf{Q}=\left[\begin{array}{cc}
\mathbf{Q}_{1} & \mathbf{0} \\
\mathbf{0} & \mathbf{Q}_{2}
\end{array}\right] .
$$

Thanks to this assumption, this channel boils down to a twousers MIMO multiple access channel. Thus, successive decoding of $\left(\mathbf{x}_{1}, \mathbf{x}_{2}\right)$ is a capacity-achieving decoding strategy and a full joint decoding is not needed.

\subsection{Problem formulation}

As in the previous section, we are interested in maximizing the mutual information between the source inputs $\left(\mathbf{x}_{1}, \mathbf{x}_{2}\right)$ and the destination outputs $\left(\mathbf{y}_{1}, \mathbf{y}_{2}\right), I\left(\mathbf{x}_{1}, \mathbf{x}_{2} ; \mathbf{y}_{1}, \mathbf{y}_{2}\right)$, over the input covariance matrices, $\mathbf{Q}_{1}$ and $\mathbf{Q}_{2}$, and the linear processing matrix at the relay, $\mathbf{G}$, under instantaneous power constraints for the source and relay input symbols. Notice that the ergodic achievable rate is $\frac{1}{2} E_{\mathcal{H}}\left[I\left(\mathbf{x}_{1}, \mathbf{x}_{2} ; \mathbf{y}_{1}, \mathbf{y}_{2}\right)\right]$. Thus we can formulate our optimization problem

$$
\begin{aligned}
& \max _{\mathbf{Q}_{1}, \mathbf{Q}_{2}, \mathbf{G}} I\left(\mathbf{x}_{1}, \mathbf{x}_{2} ; \mathbf{y}_{1}, \mathbf{y}_{2}\right) \\
& \text { s.t. }\left\{\begin{array}{l}
\mathbf{Q}_{i} \succeq \mathbf{0} \quad i=1,2 \\
\operatorname{tr}\left(\mathbf{Q}_{i}\right)=P_{i} \quad i=1,2 \\
\operatorname{tr}\left(\mathbf{R}\left(\mathbf{Q}_{1}, \mathbf{G}\right)\right)=P_{R}
\end{array}\right.
\end{aligned}
$$

Given (12) and (9) the mutual information can be written as

$$
\begin{gathered}
I\left(\mathbf{x}_{1}, \mathbf{x}_{2} ; \mathbf{y}_{1}, \mathbf{y}_{2}\right)= \\
C\left(\hat{\mathbf{H}}_{1}(\mathbf{G}) \mathbf{Q}_{1} \hat{\mathbf{H}}_{1}^{H}(\mathbf{G}) \mathbf{C}^{-1}(\mathbf{G})+\hat{\mathbf{H}}_{2} \mathbf{Q}_{2} \hat{\mathbf{H}}_{2}^{H} \mathbf{C}^{-1}(\mathbf{G})\right)
\end{gathered}
$$

where $\hat{\mathbf{H}}_{1}(\mathbf{G})=\left[\begin{array}{c}\mathbf{H}_{0} \\ \mathbf{H}_{2} \mathbf{G H}_{1}\end{array}\right]$ and $\hat{\mathbf{H}}_{2}=\left[\begin{array}{c}\mathbf{0} \\ \mathbf{H}_{0}\end{array}\right]$. In order to gain further information-theoretic insight on this optimization problem, we can use the chain rule [8] to expand the mutual information (14). Since $I\left(\mathbf{x}_{2} ; \mathbf{y}_{1} \mid \mathbf{x}_{1}, \mathbf{y}_{2}\right)=0$, we have

$$
\begin{aligned}
& I\left(\mathbf{x}_{1}, \mathbf{x}_{2} ; \mathbf{y}_{1}, \mathbf{y}_{2}\right)= \\
& \underbrace{I\left(\mathbf{x}_{1} ; \mathbf{y}_{1}\right)}_{I_{1}}+\underbrace{I\left(\mathbf{x}_{1} ; \mathbf{y}_{2} \mid \mathbf{y}_{1}\right)}_{I_{R}}+\underbrace{I\left(\mathbf{x}_{2} ; \mathbf{y}_{2} \mid \mathbf{x}_{1}\right)}_{I_{2}} .
\end{aligned}
$$

The total mutual information is the sum of three terms: the first term $I_{1}$ relates to the source transmission in the first time-slot, the second term $I_{R}$ accounts for the signal retransmitted by the relay in the second time-slot, while the third term $I_{2}$ depends on the source transmission in the second time-slot. 
Recalling (9), the terms in (15) can be evaluated as follows ${ }^{2}$

$$
\begin{aligned}
& I_{1}=C\left(\mathbf{H}_{0} \mathbf{Q}_{1} \mathbf{H}_{0}^{H}\right) \\
& I_{R}=C\left(\mathbf{H}_{2} \mathbf{G} \tilde{\mathbf{A}}\left(\mathbf{Q}_{1}\right) \tilde{\mathbf{A}}^{H}\left(\mathbf{Q}_{1}\right) \mathbf{G}^{H} \mathbf{H}_{2}^{H} \mathbf{B}^{-1}\left(\mathbf{G}, \mathbf{Q}_{2}\right)\right) \\
& I_{2}=C\left(\mathbf{H}_{0} \mathbf{Q}_{2} \mathbf{H}_{0}^{H} \mathbf{C}_{2}^{-1}(\mathbf{G})\right),
\end{aligned}
$$

where the $N \times N$ matrix $\tilde{\mathbf{A}}\left(\mathbf{Q}_{1}\right)$ is

$$
\tilde{\mathbf{A}}\left(\mathbf{Q}_{1}\right)=\mathbf{H}_{1}\left(\mathbf{Q}_{1}^{-1}+\mathbf{H}_{0}^{H} \mathbf{H}_{0}\right)^{-\frac{1}{2}},
$$

and the $N \times N$ matrix $\mathbf{B}\left(\mathbf{G}, \mathbf{Q}_{2}\right)$ accounts for all the interference and noise on the channel between the relay and the destination in the second time-slot:

$$
\mathbf{B}=\sigma^{2} \mathbf{I}+\sigma^{2} \mathbf{H}_{2} \mathbf{G G}^{H} \mathbf{H}_{2}^{H}+\mathbf{H}_{0} \mathbf{Q}_{2} \mathbf{H}_{0}^{H} .
$$

\subsection{An iterative solution}

The optimization problem (13) is not convex. However, if we fix either $\left(\mathbf{Q}_{1}, \mathbf{Q}_{2}\right)$ or $\mathbf{G}$, the resulting problem is convex in the remaining variable. Therefore, similarly to the previous section, we can devise an iterative procedure that alternates between the optimization over $\mathbf{G}$ fixed $\left(\mathbf{Q}_{1}, \mathbf{Q}_{2}\right)$ and the optimization over $\left(\mathbf{Q}_{1}, \mathbf{Q}_{2}\right)$ fixed $\mathbf{G}$. Again, absolute convergence to an optimal solution for this algorithm cannot be proved, since the power constraint on the relay input symbol depends upon both $\mathbf{Q}_{1}$ and $\mathbf{G}$. Nevertheless, if the problem is well- conditioned the algorithm has shown in practice rapid convergence (around five iterations) to a unique solution.

Let us first fix $\mathbf{G}$. The resulting optimization problem over $\left(\mathbf{Q}_{1}, \mathbf{Q}_{2}\right)$ is convex. As we did in the previous section, for the sake of our algorithm, the power constraint on the relay input symbol can be ignored. Resorting to the mutual information expression found in (14), our statement of the problem is

$$
\begin{aligned}
& \max _{\mathbf{Q}_{1}, \mathbf{Q}_{2}} C\left(\hat{\mathbf{H}}_{1} \mathbf{Q}_{1} \hat{\mathbf{H}}_{1}^{H} \mathbf{C}^{-1}+\hat{\mathbf{H}}_{2} \mathbf{Q}_{2} \hat{\mathbf{H}}_{2}^{H} \mathbf{C}^{-1}\right) \\
& \text { s.t. }\left\{\begin{array}{l}
\mathbf{Q}_{i} \succeq \mathbf{0} \quad i=1,2 \\
\operatorname{tr}\left(\mathbf{Q}_{i}\right)=P_{i} \quad i=1,2
\end{array} .\right.
\end{aligned}
$$

This problem is identical to finding the optimal input covariance matrices for a two-users MIMO-MAC with channels $\hat{\mathbf{H}}_{1}$ and $\hat{\mathbf{H}}_{2}$ and noise covariance matrix $\mathbf{C}$. The solution to this problem is the iterative-waterfilling algorithm proposed in [9].

Let us now fix $\mathbf{Q}_{1}, \mathbf{Q}_{2}$. In this case, it is better to use the mutual information expansion found in (16), since it fully

\footnotetext{
${ }^{2}$ Notably, we could have obtained (16) also using the properties of the determinant of block matrices. Anyway, we preferred to present this result according to a more insightful information-theoretic approach.
}

reveals the role played by $\mathbf{G}$ in this scheme. Obviously, $\mathbf{G}$ affects only the second time-slot terms. Focusing on these last two terms, it appears to be very hard to find an analytical expression for a matrix $\mathbf{G}$ capable of maximizing jointly the relay mutual information term $I_{R}$ and the source mutual information term $I_{2}$. However, we observe that, as further detailed in Sec. 4, under appropriate conditions (namely a sufficiently good channel between source and relay) the term $I_{2}$ can be neglected without relevant performance loss. It can be proved that, with a further manipulation of the expression of $I_{R}$, the optimization problem boils down to a form similar to (6), so we can use the same arguments discussed in the previous section. In particular, if we set $\mathbf{G}=\tilde{\mathbf{V}}_{2} \tilde{\mathbf{D}}_{g} \tilde{\mathbf{U}}_{A}^{H}$, where $\tilde{\mathbf{D}}_{g}$ is diagonal, $\tilde{\mathbf{V}}_{2}$ is the matrix of the right eigenvectors of $\tilde{\mathbf{H}}_{2}=\left(\mathbf{I}+\frac{1}{\sigma^{2}} \mathbf{H}_{0} \mathbf{Q}_{2} \mathbf{H}_{0}^{H}\right)^{-\frac{H}{2}} \mathbf{H}_{2}$ and $\tilde{\mathbf{U}}_{A}$ is the matrix of the left eigenvectors of $\tilde{\mathbf{A}}$, it can be shown that the maximization of $I_{R}$ over $\mathbf{G}$ leads to

$$
\begin{gathered}
\left|\tilde{g}_{r}\right|^{2}=\frac{1}{\tilde{\lambda}_{A, r}^{2}+\sigma^{2}}\left[\tilde{f}\left(\mu ; \tilde{\eta}_{r}\right)-\frac{\sigma^{2}}{\tilde{\lambda}_{2, r}^{2}}\right]^{+} \\
\tilde{f}\left(\mu ; \tilde{\eta}_{r}\right)=\sqrt{\left(\frac{\tilde{\eta}_{r}}{2}\right)^{2}+\frac{\tilde{\lambda}_{A, r}^{2}+\sigma^{2}}{a_{r r}+\sigma^{2}} \tilde{\eta}_{r} \mu^{2}}-\frac{\tilde{\eta}_{r}}{2},
\end{gathered}
$$

where $\tilde{\lambda}_{A, r}$ and $\tilde{\lambda}_{2, r}$ are the $r$-th singular value of $\tilde{\mathbf{A}}$ and $\tilde{\mathbf{H}}_{2} ; \tilde{g}_{r}$ is the $r$-th diagonal element of the matrix $\tilde{\mathbf{D}}_{g} ; a_{r r}$ is the nonnegative $r$-th diagonal element of the semi-definite positive matrix $\tilde{\mathbf{U}}_{A}^{H} \mathbf{H}_{1} \mathbf{Q}_{1} \mathbf{H}_{1}^{H} \tilde{\mathbf{U}}_{A}$, and $\tilde{\eta}_{r}=\frac{\tilde{\lambda}_{A, r}^{2}}{\tilde{\lambda}_{2, r}^{2}}$. The value of $\mu$ is chosen as to satisfy the power constraint on the relay input symbol $\sum_{r=1}^{N}\left(a_{r r}+\sigma^{2}\right)\left|\tilde{g}_{r}\right|^{2}=P_{R}$.

Following from this results, we can finally detail our algorithm: (a) solve the optimization problem (19) for $\left(\mathbf{Q}_{1}\right.$, $\mathbf{Q}_{2}$ ) keeping $\mathbf{G}$ fixed; $(b)$ solve the the problem of optimizing $I_{R}$ over $\mathbf{G}$ keeping $\left(\mathbf{Q}_{1}, \mathbf{Q}_{2}\right)$ fixed. Notice that the last iteration of the algorithm has to be the optimization of $I_{R}$ over $\mathbf{G}$, since this guarantees the enforcement of all the constraints in the original problem (13).

\section{SIMULATION RESULTS}

In this section, we assume that the relay is located on a line between the source and the destination, at a normalized distance $d \in[0,1]$ from the source and $(1-d)$ from the destination. It follows that, assuming a path-loss exponent of 4 , the channel matrix $\mathbf{H}_{i}$ has entries distributed as $\mathcal{C N}\left(0, d_{i}^{-4}\right)$, where $d_{1}=d, d_{2}=(1-d)$ and $d_{0}=1$. Moreover, for lack of space, we focus on the collaborative scenario (similiar results hold for the multi-hop case). As far as the power constraints are concerned, we fix $P_{1}=1$ and $P_{2}=P_{R}=\frac{1}{2}$, so as to satisfy a per-slot sum-power constraint $P_{2}+P_{R}=1$ and obtain fair performance comparison. 


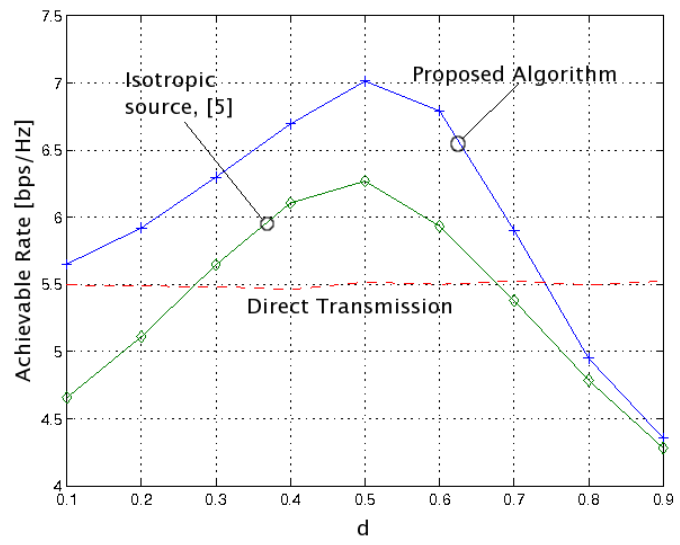

Fig. 2. Achievable rates of different communication schemes for the cooperative system with $\frac{1}{\sigma^{2}}=5 d B, N=$ 3 .

The achievable rates of different algorithms for the cooperative scenario are depicted in fig. 2 versus the distance $d$ for $\frac{1}{\sigma^{2}}=5 d B$ and a number of antennas at each node $N=3$. In particular, both the technique presented in [5] (that assumes an isotropic covariance matrix $\mathbf{Q}=$ $\left.N^{-1} \mathbf{I}\right)$ and the proposed method are compared to the reference performance of direct transmission (between source and destination). In this case, the proposed algorithm outperforms both direct transmission (by up to $1.5 \mathrm{bps} / \mathrm{Hz}$ ) and the scheme of [5] (by up to $0.8 \mathrm{bps} / \mathrm{Hz}$ ) for almost every value of $d$.

Finally, we would like to comment on the choice of neglecting the term $I_{2}$ while optimizing for $\mathbf{G}$ in Sec. 3.3. Fig. 3 shows $I_{2}$ versus $d$ for the proposed scheme, along with the upper bound $I_{2} \leq C\left(\frac{1}{\sigma^{2}} \mathbf{H}_{0} \mathbf{Q}_{2} \mathbf{H}_{0}^{H}\right)=I_{2 u b}$. It is seen that for $d \leq 0.4$ (i.e., for a sufficiently good channel between source and relay), there is no optimality loss due to $I_{2}$. However, for $d \geq 0.4$ it is envisaged that a joint optimization of $I_{2}$ and $I_{R}$ over $\mathbf{G}$ might bring performance benefits.

\section{CONCLUSIONS}

In this paper multi-hop and cooperative MIMO Amplifyand-Forward relay systems were considered. The problem of maximizing the achievable rate over the source covariance matrices and linear processing at the relay has been formulated assuming full CSI at each node. A sub-optimal iterative algorithm has been devised, exploiting the convexity properties of the problem.

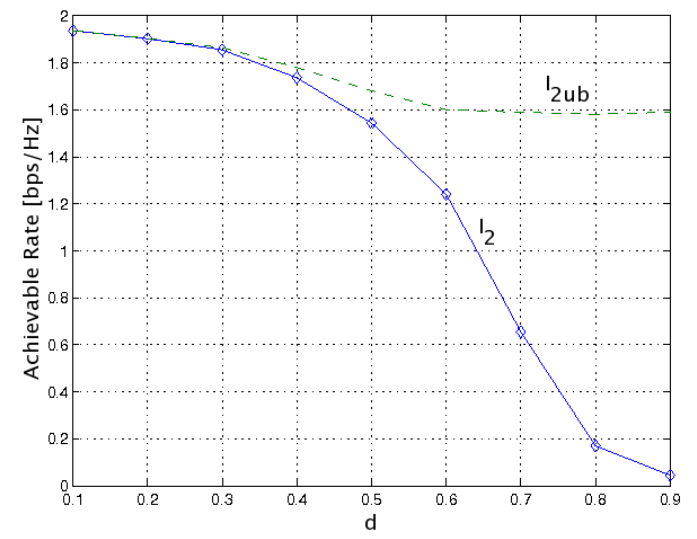

Fig. 3. Rate component $I_{2}$ and upper bound $I_{2 u b}$ as a function of $d$ for $\frac{1}{\sigma^{2}}=5 d B, N=3$.

\section{REFERENCES}

[1] T. M. Cover and A. A. E. Gamal, "Capacity theorems for the relay channel," IEEE Trans. Inform. Theory, vol. 25 , no. 6 , pp. 572-584, Sept. 1979.

[2] J. N. Laneman, D. N. C. Tse, and G. W. Wornell, “Cooperative diversity in wireless networks: efficient protocols and outage behaviour," IEEE Trans. Inform. Theory, vol. 50, no. 6, pp. 3062-3080, Dec. 2004.

[3] R. U. Nabar, H. Bolcskei, and F. W. Kneubuhler, "Fading relay channels: Performance limits and space-time signal design," IEEE J. Select. Areas Commun., vol. 22, no. 6, pp. 1099-1109, June 2004.

[4] B. Wang, J. Zhang, and A. Host-Madsen, "On the capacity of MIMO relay channels," IEEE Trans. Inform. Theory, vol. 51, no. 1, pp. 29-43, Jan. 2005.

[5] O. Munoz, J. Vidal, and A. Agustin, "Non-regenerative MIMO relaying with channel state information," in Proc. ICASSP'05, vol. 3, Mar. 2005.

[6] I. E. Telatar, "Capacity of multi-antenna gaussian channels," Eur. Trans. Telecommun., vol. 10, pp. 585-595, Nov. 1999.

[7] D. Bertsekas, Nonlinear Programming. Athena Scientific, 1999.

[8] T. M. Cover and J. A. Thomas, Elements of Information Theory. Wiley Interscience, 1991.

[9] W. Yu, W. Rhee, S. Boyd, and J. Cioffi, "Iterative waterfilling for gaussian vector multiple access channels," IEEE Trans. Inform. Theory, vol. 50, pp. 145-152, Jan. 2004. 